Research Paper

\title{
Molecular and functional characterization of two malic enzymes from Leishmania parasites
}

\author{
Lucila Giordana $^{\mathrm{a}, 1}$, Máximo Hernán Sosa ${ }^{\mathrm{b}, 1}$, Alejandro E. Leroux ${ }^{\mathrm{c}}$, Elkin F. Rodas Mendoza ${ }^{\mathrm{d}}$, \\ Patricia Petray ${ }^{\mathrm{e}}$, Cristina Nowicki ${ }^{\mathrm{a}, *}$

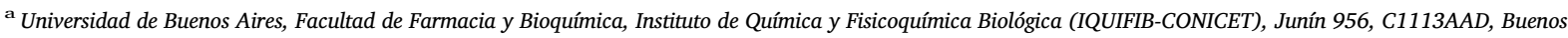 \\ Aires, Argentina \\ ${ }^{\mathrm{b}}$ Universidad de Buenos Aires, Facultad de Farmacia y Bioquímica, Instituto de Investigaciones Farmacológicas en alianza estratégica con UBA-CONICET (ININFA) Junín \\ 956, C1113AAD, Buenos Aires, Argentina \\ ${ }^{\mathrm{c}}$ Instituto de Investigación en Biomedicina de Buenos Aires (IBioBA)-CONICET -Partner Institute of the Max Planck Society, Buenos Aires C1415FQD, Argentina \\ d Universidade Estadual Paulista (Unesp), Faculdade de Ciências Agrárias e Veterinárias, Jaboticabal, Brazil \\ e Universidad de Buenos Aires, Instituto de Microbiología y Parasitología Médica (IMPaM-CONICET), Paraguay 2155, C1121ABG, Buenos Aires, Argentina
}

\section{A R T I C L E I N F O}

\section{Keywords:}

Leishmania

Malic enzymes

NADPH production

Redox metabolism

\begin{abstract}
A B S T R A C T
Leishmania parasites cause a broad spectrum of clinical manifestations in humans and the available clinical treatments are far from satisfactory. Since these pathogens require large amounts of NADPH to maintain intracellular redox homeostasis, oxidoreductases that catalyze the production of NADPH are considered as potential drug targets against these diseases. In the sequenced genomes of most Leishmania spp. two putative malic enzymes (MEs) with an identity of about 55\% have been identified. In this work, the ME from $L$. major (LmjF24.0770, Lmj_ME-70) and its less similar homolog from L. mexicana (LmxM.24.0761, Lmex_ME-61) were cloned and functionally characterized. Both MEs specifically catalyzed NADPH production, but only Lmex_ME61 was activated by L-aspartate. Unlike the allosterically activated human ME, Lmex_ME-61 exhibited typical hyperbolic curves without any sign of cooperativity in the absence of L-aspartate. Moreover, Lmex_ME-61 and Lmj_ME-70 differ from higher eukaryotic homologs in that they display dimeric instead of tetrameric molecular organization. Homology modeling analysis showed that Lmex_ME-61 and Lmj_ME-70 notably differ in their surface charge distribution; this feature encompasses the coenzyme binding pockets as well. However, in both isozymes, the residues directly involved in the coenzyme binding exhibited a good degree of conservation. Besides, only Lmex_ME-61 and its closest homologs were immunodetected in cell-free extracts from L. mexicana, L. amazonensis and L. braziliensis promastigotes. Our findings provide a first glimpse into the biochemical properties of leishmanial MEs and suggest that MEs could be potentially related to the metabolic differences among the species of Leishmania parasites.
\end{abstract}

\section{Introduction}

Malic enzymes (MEs) are widely distributed throughout all kingdoms of life. These enzymes catalyze the reversible oxidative decarboxylation of L-malate to yield pyruvate and $\mathrm{CO}_{2}$ with the reduction of either $\mathrm{NADP}^{+}$or $\mathrm{NAD}^{+}$, according to the specificity towards the coenzyme. MEs share a relatively high sequence relatedness, which results in highly conserved active sites and structure topology, with most of them exhibiting a tetrameric organization (dimer of dimers). In mammals, MEs have been divided in three groups: the cytosolic $\mathrm{NADP}^{+}$-dependent ME (c-NADP-ME), and the two mitochondrial isoforms, the $\mathrm{NADP}^{+}$-dependent ME (m-NADP-ME) and that NAD $(\mathrm{P})^{+}$dependent ME (m-NAD(P)-ME) [1,and references therein]. While the cytosolic ME is mainly involved in biosynthetic processes and oxidative stress defense, the mitochondrial isoforms are mostly implicated in energy transduction [2]. In man, the m-NAD(P)-ME differs from the other isoforms in that it is allosterically activated by fumarate [3].

It is well known that among the members of the family Trypanosomatidae, trypanosomes and the about 20 species of the genus Leishmania that produce different diseases in man are comprised. These pathogens consume large amounts of NADPH for the detoxification of oxygen and nitrosative species generated during the parasite-host

\footnotetext{
* Corresponding author.

E-mail address: cnowicki@qb.ffyb.uba.ar (C. Nowicki).

${ }^{1}$ These authors contributed equally.
} 
interactions. Notably, NADPH represents the only reducing power which guarantees the continuous regeneration of trypanothione. This unique low molecular mass dithiol is essential for the maintenance of intracellular redox homeostasis and survival of trypanosomes and Leishmania parasites within their insect vectors and mammalian hosts [for review see 4,5]. Besides, these organisms also depend on NADPH for other key processes such as anabolism [6], synthesis of DNA precursors or the ferrous ion uptake. The latter is particularly critical for the cellular remodeling of leishmanial promastigotes into amastigotes [for review see 7]. Based on the key roles of NADPH in the survival of these pathogenic protozoa, the two dehydrogenases of the pentose phosphate pathway have been proposed as potential drug targets for clinical treatments of the diseases caused in man by trypanosomes and Leishmania parasites [8]. The latter route starts from glucose 6-phosphate which is formed by phosphorylation of glucose by hexokinase, in the first reaction-step of the glycolytic pathway. However, leishmanial amastigotes exhibit much reduced rates of glucose uptake, which results in a glucose-sparing metabolism. This switch to more economical metabolic processes occurs regardless of the availability of glucose or other carbon sources in the medium [9]. In this context, MEs might represent the second most important source of NADPH for the intracellular stage of this parasite [for a review see 10]. In line with this hypothesis, the cytosolic ME of T. brucei is essential for survival when the expression of glucose- 6 phosphate dehydrogenase is repressed [11].

Genome sequencing of Leishmania spp. has revealed that, with the exception of $L$. major, parasites such as $L$. mexicana, $L$. braziliensis, $L$. donovani and $L$. infantum exhibit two different gene copies encoding putative MEs (http://www.genedb.org). The two potential isozymes display a sequence identity of about $55 \%$. However, no leishmanial ME has been purified to protein homogeneity or functionally characterized so far.

Keeping in mind that most Leishmania spp. are the causative agents of a broad spectrum of clinical manifestations in humans, which, if untreated, lead to severe damaging, morbility and death, the aim of the present study was to evaluate the biochemical properties of the two putative ME isoforms predicted to be operative in Leishmania spp. To this end, we selected the putative ME from L. major and the less similar homolog from L. mexicana. Our results revealed that Leishmania parasites exhibit two functional ME isozymes which specifically catalyze NADPH production, but differ in their kinetic properties, structure topology and appear to exhibit a species-specific expression pattern.

\section{Materials and methods}

\subsection{Organisms and culture}

L. mexicana and $L$. major promastigotes were grown as previously described [12], while L. braziliensis (strain MHOM/BR/1975/M2903) and L. amazonensis (strain MHOM/BR/1973/M2269) promastigotes were cultured in $25-\mathrm{ml}$ flasks at $26^{\circ} \mathrm{C}$ in Schneider's Insect Medium, $\mathrm{pH}$ 7.2, (Sigma, St Louis, MO, USA) supplemented with $10 \%$ inactivated fetal bovine serum, $100 \mathrm{IU} / \mathrm{ml}$ penicillin, $100 \mu \mathrm{g} / \mathrm{ml}$ streptomycin, and $2 \mathrm{mM}$ glutamine. Cultures were routinely maintained by weekly passages.

\subsection{Cloning and expression of putative $M E$ isozymes from $L$. major and $L$.} mexicana

Total DNA was isolated from L. major and L. mexicana promastigotes as previously described [13]. The coding sequences of the putative $M E s$ from L. major (LmjF24.0770, Lmj_ME-70) and $L$. mexicana (LmxM.24.0761, Lmex_ME-61) were amplified by PCR using genomic DNA as template and Phusion DNA polymerase (New England Biolabs). Specific primers were designed on the basis of the predicted open reading frames (ORFs) in the genome project database (http://www. genedb.org): Lmj_ME7O-fw 5'- catatgtttgccaagtcgctg-3'; Lmj_ME7O-rev
5'- gaattcttagcgaatcaactccttctcc-3'; Lmex_ME61-fw 5'- catatgtccctcggaa acgatctgc-3'; Lmex_ME61-rev 5'gaattctcacaggaggctggcgtcc-3'. The forward primers contained an NdeI restriction site (underlined), whereas the reverse primers contained an EcoRI restriction site (underlined). PCR settings were $5 \mathrm{~min}$ at $95{ }^{\circ} \mathrm{C}$ and 25 cycles involving denaturation at $95{ }^{\circ} \mathrm{C}$ for $45 \mathrm{~s}$, annealing at $60^{\circ} \mathrm{C}$ and $58{ }^{\circ} \mathrm{C}$ for $45 \mathrm{~s}$ for Lmex_ME-61, and $L m j_{-} M E-70$ respectively, and extension at $72{ }^{\circ} \mathrm{C}$ for $90 \mathrm{~s}$. Besides, a final extension step at $72{ }^{\circ} \mathrm{C}$ for $10 \mathrm{~min}$ was performed. In each of the two reactions, a single fragment $(\cong 1.8 \mathrm{~kb})$ was amplified. Upon agarose gel purification, the PCR products were cloned into the pGEM-T easy vector and the ligated DNA fragments were fully sequenced. The nucleotide sequences coding for both MEs were subcloned into the pET28a $^{+}$expression vector (Novagen, Darmstadt, Germany), thus generating pET28-Lmex_ME-61 and pET28-Lmj_ME-70. The 5'-ends of the $L$. major and $L$. mexicana genes were similarly extended with a nucleotide sequence encoding a 6 xHis-tag and a thrombin cleavage site. pET28-Lmex_ME-61 and an expression plasmid containing the coding sequence for pGro7 were used to transform Escherichia coli BL21-CodonPlus (DE3) RIL, whereas pET28-Lmj_ME-70 was introduced into $E$. coli Rosetta (DE3) pLysS. Subsequently, both strains of transformed bacteria were grown in LB medium supplemented with $19.9 \mu \mathrm{g} \mathrm{ml} \mathrm{l}^{-1}$ and $34 \mu \mathrm{g} \mathrm{ml}^{-1}$ of chloramphenicol, respectively, as well as with $30 \mu \mathrm{g} \mathrm{ml}^{-1}$ kanamycin at $37^{\circ} \mathrm{C}$, until an $\mathrm{OD}_{600 \mathrm{~nm}}$ of 0.6 was reached. In the case of Lmj_ME-70, the expression was initiated by adding isopropyl- $\alpha$-D-thiogalactopyranoside (IPTG) to a final concentration of $0.2 \mathrm{mM}$, and the cultures were further grown at $20^{\circ} \mathrm{C}$, for $4 \mathrm{~h}$. Instead, the production of Lmex_ME-61 was induced by adding IPTG to a final concentration of $0.1 \mathrm{mM}$ and arabinose $0.49 \mathrm{mg} \mathrm{ml}^{-1}$. The cultures were further grown at $20^{\circ} \mathrm{C}$, for approximately $16 \mathrm{~h}$.

Bacteria were harvested by centrifugation and the pellets suspended in $20 \mathrm{mM} \mathrm{HCl}$-Tris buffer, $\mathrm{pH} \mathrm{7.9,} \mathrm{containing} 5 \mathrm{mM}$ imidazole and $500 \mathrm{mM} \mathrm{NaCl}$. Upon sonication, the recombinant proteins were purified by affinity chromatography on a $\mathrm{Ni}^{2+}$-nitrilotriacetic column (Qiagen, Germany), and the fractions with enzymatic activity were pooled and immediately desalted in a PD-10 column (GE-Healthcare) equilibrated with $75 \mathrm{mM}$ Triethanolamine (TEA) buffer, $\mathrm{pH}$ 7.4. The protein homogeneity of both recombinant enzymes was analyzed by SDS-PAGE [14]. The method of Bradford was used to determine protein concentration; bovine serum albumin was used as standard [15]. Moreover, to evaluate the molecular organization of the two ME isozymes in native conditions, Lmj_ME-70 and Lmex_ME-61 were subjected to gel-filtration chromatography on a Sephacryl-S200 h column equilibrated with $75 \mathrm{mM}$ TEA buffer, $\mathrm{pH}$ 7.4. The elution was monitored at $280 \mathrm{~nm}$, and the molecular-mass markers used to calibrate the column were: cystathionine $\beta$ synthase (156.8 kDa), phosphoenolpyruvate carboxykinase (103 kDa), tyrosine aminotransferase (90 kDa), hemoglobin (68 kDa); and ovoalbumin (45 kDa).

\subsection{Kinetic characterization of the $M E$ isozymes from $L$. major and $L$. mexicana}

The activities of the recombinant ME isozymes were assayed spectrophotometrically at $37^{\circ} \mathrm{C}$, in $75 \mathrm{mM}$ TEA buffer, $\mathrm{pH}$ 7.4. The oxidative decarboxylation of malate was examined in the presence of $0.5 \mathrm{mM}$ $\mathrm{MnCl}_{2}, 0.5 \mathrm{mM} \mathrm{NADP}^{+}$and $5 \mathrm{mM}$ L-malate. The ability to catalyze pyruvate carboxylation was tested in $100 \mathrm{mM}$ acetate buffer, $\mathrm{pH} 5.4$, containing $0.5 \mathrm{mM} \mathrm{MnCl}_{2}, 0.5 \mathrm{mM} \mathrm{NADPH}, 5 \mathrm{mM} \mathrm{NaHCO}_{3}$ and $50 \mathrm{mM}$ pyruvate. The reactions were initiated by the addition of the corresponding recombinant enzyme and the activities were determined by following the absorbance increase (due to NADPH production) or decrease (due to NADPH consumption) at $340 \mathrm{~nm}$. The kinetic parameters were determined by varying the concentration of the tested substrate while the concentrations of the other substrates were kept at saturating levels. One unit of ME activity was defined as the amount of enzyme that catalyzes the production of $1 \mu \mathrm{mol}$ of NADPH per minute. The kinetic constants are the means of at least four determinations and were 
calculated by fitting the initial rates to the Michaelis-Menten Eqs. (1) and (2) by non-linear regression using GraphPad Prism 5.01.

$\mathrm{v}_{\mathrm{i}}=k_{\mathrm{cat}}[\mathrm{S}] / K_{\mathrm{m}}+[\mathrm{S}]$

$1 / v=K_{\mathrm{m}} / V_{\max }(1 /[\mathrm{S}])+1 / V_{\max }$

Turnover numbers $\left(\mathrm{k}_{\text {cat }} \mathrm{s}^{-1}\right)$ were calculated using the molecular mass of one subunit of each ME. To examine whether leishmanial ME isozymes were subjected to allosteric modulation, the activities in the direction of malate decarboxylation were determined in the presence and absence of selected effectors. The reaction mixture described above was used, except that the final concentration of L-malate was $0.2 \mathrm{mM}$. The effects of potential effectors such as L-aspartate $(5 \mathrm{mM})$, fumarate ( $5 \mathrm{mM})$, acetyl-CoA $(5 \mu \mathrm{M}), 2$-oxoglutarate $(0.5 \mathrm{mM})$, and L-glutamate $(0.5 \mathrm{mM})$ were assayed at the final concentrations indicated in parentheses.

\subsection{Western blot analyses}

Polyclonal antisera against the two purified recombinant MEs (Lmj_ME-70 and Lmex_ME-61) were raised in mice. To examine the specificity of each antiserum, various amounts of the recombinant isozymes were subjected to SDS-PAGE, and transferred onto nitrocellulose membranes, which were subsequently incubated with either the polyclonal anti-Lmj_ME-70 (1:2000) or anti-Lmex_ME-61 (1:5000). The immunoconjugates were developed by using ECL Plus Western Blotting substrate (Pierce), following the manufacturer's protocols. The chemiluminescent signal was either automatically detected by ImageQuant LAS500 or by exposing the membranes to X-ray films. On the other hand, cell-free extracts of promastigotes $\left(\cong 50 \times 10^{6}\right.$ cells) from $L$. mexicana, $L$. braziliensis and $L$. amazonensis were obtained by three cycles of freezing and thawing in $50 \mathrm{mM}$ HCl-Tris buffer, $\mathrm{pH} 8$, containing $1 \mathrm{mM}$ EDTA, $1 \mathrm{mM}$ 1,4-dithiothreitol, 0.1\% Triton X-100, $5 \mu \mathrm{M}$ leupeptin, $1 \mu \mathrm{M}$ phenylmethanesulphonyl fluoride, $2 \mu \mathrm{M}$ pepstatin $\mathrm{A}$ and $0.5 \mathrm{mM}$ Na-tosyl-Lys-chloromethyl ketone. The proteins contained in the total extracts were resolved by SDS-PAGE on $8.5 \%$ polyacrylamide gels and transferred by electro-blotting to nitrocellulose membranes. Subsequently, the immobilized proteins were incubated with the polyclonal mouse antiserum generated against each of the recombinant MEs. The immunoconjugates were developed using antimouse IgG antibodies coupled to peroxidase antibody and by following exactly the same protocols as described above.

\subsection{Phylogenetic analyses}

Sequences assigned to $21 \mathrm{MEs}$ from the order Kinetoplastea were retrieved from GENEdb (www.genedb.org) and the multiple sequence alignment was conducted using the default parameters of the ClustalW software (http://www.ch.embnet.org/software/ClustalW.html). This alignment was used to build the phylogenetic tree with TREEFINDER. Maximum-likelihood phylogenies were estimated using TREEFINDER; the reconstruction of the phylogenetic tree and the estimation of bootstrap proportions were achieved by using default parameters [16]. The ME isozymes from Bodo saltans, an organism considered the closest nonparasitic relative of trypanosomatids [17] were used as outgroups. The accession numbers of the sequences used in the phylogenetic analysis are as follows: B. saltans (BSAL_85840, CUG78626.1; BSAL_85845, CUG786 38.1), L. braziliensis (LbrM.24.0780, XP_001565344.2; LbrM.24.0790, XP_001565345.2), L. donovani (LdBPK 240780.1, XP_003861168.1; LdBPK 240790.1, XP_003861169.1), L. infantum (LinJ.24.0780, XP_001 465906.1; LinJ.24.0790, XP_001465907.1), L. major (LmjF.24.0770, XP_001683592.1), L. mexicana (LmxM.24.0761, XP_003875889.1; LmxM.24.0770, XP_003875890.1), T. brucei (Tb927.11.5440, XP_828619.1; Tb927.11.5450, XP_828620.1), T. gambiense (Tbg9 72.11.6140, XP_011779760.1; Tbg972.11.6150, XP_011779761.1), $T$. congolense (TcIL3000.11.5680, CCC95154.1; TcIL3000.11.5690, CCC
95155.1) T. cruzi (TcCLB.505183.20, XP_814409.1; TcCLB.505183.30, accession number XP_814410.1) and $T$. vivax (TvY486_1105620, CCC53078.1; TvY486_1105630, CCC53079.1).

\subsection{Homology modeling of Lmj_ME-70 and Lmex_ME-61}

As a first approach BLAST analysis was performed using the protein data bank. The outcomes from this search showed that among the enzymes with known crystal structures the pigeon ME exhibited a slightly higher overall sequence identity to Lmj_ME-70 and Lmex_ME-61 than the human mitochondrial NAD(P) and cytosolic NADP MEs $\cong \simeq 40 \%$ vs $\cong$ 38-36\%). Subsequently, the 3-D structures of the NADP-dependent ME from pigeon liver in a quaternary complex with $\mathrm{NADP}^{+}$, oxalate, and $\mathrm{Mn}^{2+}$ (PBD 1GQ2) in addition to the pentary complex (including fumarate) of the human mitochondrial $\mathrm{NAD}(\mathrm{P})^{+}$-dependent ME (1PJ3) were used as templates to construct the structure-based amino acid sequence alignment by using the ESPript 3.0 web tool (http://espript. ibcp.fr/ESPript/ESPript/) [18]. Thereafter, the pigeon ME structure (PBD 1GQ2) was selected as template to build homology models of Lmj_ME-70 (LmjF24.0770) and Lmex_ME-61 (LmxM.24.0761). The 3-D structural models were generated with MODELLER 9.17. This software produced five models for each ME. Those with the lowest DOPE scores were selected. Next, further full-atomic refinement based on energy minimization of the best model generated for Lmj_ME-70 and Lmex_ME61 was performed by using the ModRefiner algorithm at http:// zhanglab.ccmb.med.umich.edu/ModRefiner [19]. The quality of the resulting models was assessed by means of Procheck Ramachandran Plot [20] and Verify3D [21]. The 3-D models of leishmanial ME isoforms were also analyzed using the COFACTOR platform for structurebased multiple-level protein function predictions at http://zhanglab. ccmb.med.umich.edu/COFACTOR [22]. Model images were generated by using UCSF Chimera 1.11.2 (https://www.cgl.ucsf.edu/chimera/) [23]. The residues which interact with $\mathrm{NADP}^{+}$were visualized by using LIGPLOT PLUS v.1.4.5 (http://www.ebi.ac.uk/thornton-srv/software/ LIGPLOT/) [24]. Besides, the evolutionary conservation of amino acid positions among relevant homologs was examined using ConSurf server (http://consurf.tau.ac.il) [25].

\section{Results}

\subsection{Comparative sequence analysis of leishmanial ME isozymes}

The $L$. major genome exhibits a single gene coding for a putative ME (LmjF24.0770), while two gene copies allocated contiguously in the same chromosome encode potentially functional MEs in the genomes of L. mexicana (LmxM.24.0761 and LmxM.24.0770), L. braziliensis (LbrM.24.0780 and LbrM.24.0790), L. donovani (LdBPK 240780.1 and $L d B P K$ 240790.1) and L. infantum (LinJ.24.0780 and LinJ.24.0790). The protein sequences encoded by $L m j F 24.0770$, $L m x M .24 .0770$, LinJ.24.0790, LdBPK 240790.1 and LbrM.24.0790 are virtually identical (I $\cong 96 \%$ ). Also, the second set of genes referred to as $L m x M .24 .0761$, LbrM.24.0780, LdBPK240780.1 and LinJ.24.0780 code for practically identical proteins ( $\mathrm{I} \cong 98 \%$ ). However, the comparison of the amino acid sequences derived from these two sets of leishmanial genes evidenced the plausible presence of two potential ME isoforms with a sequence identity of about $56 \%$. Phylogenetic analysis performed using Bodo saltans MEs as a non-trypanosomatid outgroups indicated that the putative isoforms from Leishmania parasites fall in two different clades. The sequences encoded by LmxM.24.0761, LinJ.24.0780, LdBPK240780.1 and LbrM.24.0780 clustered with the cytosolic MEs from T. cruzi and T. brucei, whereas the second group of leishmanial MEs (LinJ.24.0790, LbrM.24.0790, LmxM.24.0770 and LmjF24.0770) clustered within the same clade as the mitochondrial orthologs from $T$. cruzi and T. brucei. In good agreement with the phylogenetic relationship, the putative leishmanial MEs which clustered with the cytosolic $T$. cruzi and T. brucei isoforms also shared the predicted $\mathrm{pI}$ values with the 


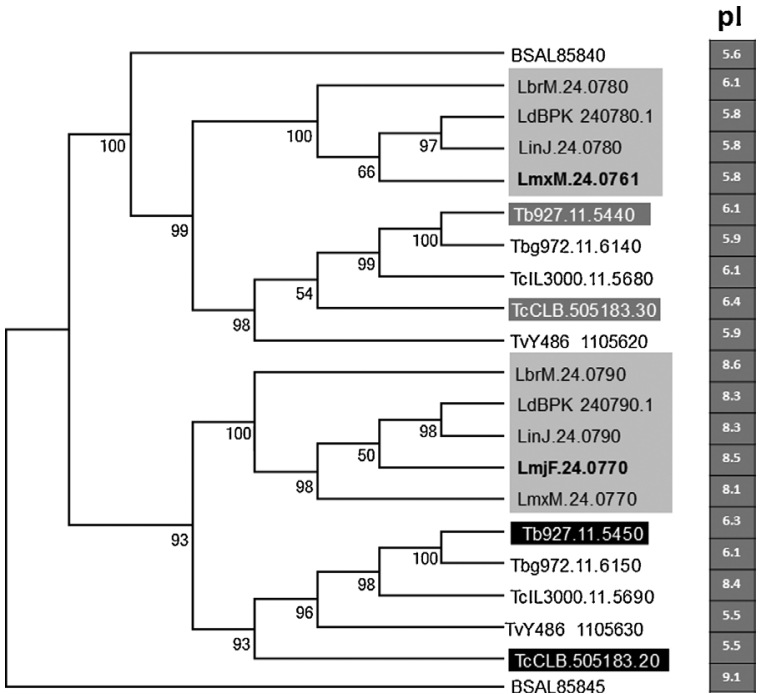

Fig. 1. Maximum-Likelihood phylogeny of MEs from kinetoplastids. The reconstruction of the phylogenetic tree and the estimation of bootstrap proportions were achieved by using default parameters of the TREEFINDER software (see Section 2). The numbers shown below subtending branches represent bootstrap values (1000 replicates, support threshold $\geq 50 \%$ ). The tree is unrooted and $B$. saltans homologs were used as outgroups. Leishmanial MEs are depicted on a gray background, and the enzymes characterized in this work are indicated in bold lettering. The cytosolic and mitochondrial MEs from $T$. cruzi and T. brucei [26] are indicated on a dark gray background and white lettering, and black background and white lettering, respectively. On the right side of the figure, a panel with the predicted pIs values is included. Abbreviations and sequence accession numbers are indicated in Section 2

latter isozymes (Fig. 1). By contrast, this parameter was not so strictly conserved among the counterparts more closely related to the mitochondrial T. cruzi and T. brucei MEs (Fig. 1). Opposed to the pI values, the predicted molecular masses for the putative MEs from the genus Leishmania (L. major, L. mexicana, L. braziliensis and L. infantum) were almost identical.

\subsection{Functional characterization of the two putative isozymes of $M E$ from Leishmania parasites}

\subsubsection{Cloning and functional expression of the recombinant Lmex_ME-61 and Lmj_ME-70}

To determine the biochemical properties of the two potential isozymes of leishmanial MEs, as a first approach, we intended to clone the genes that code for LmxM.24.0761 and LmxM.24.0770. However, despite repeated efforts, the ORF corresponding to LmxM.24.0770 could not be amplified by PCR reactions. Therefore, the closest homolog
LmjF24.0770 from L. major was selected for ongoing studies. Subsequently, as described in Section 2, the sequences encoding LmjF24.0770 and LmxM.24.0761 were amplified, cloned into the pET28 vector and functionally expressed in $E$. coli. To facilitate protein purification, a short affinity tag consisting of $6 x$ His and a thrombin cleavage site was added to the N-terminus of each of the recombinant enzymes. The recombinant $L$. major and $L$. mexicana MEs were purified by a single step of affinity chromatography onto a $\mathrm{Ni}^{2+}$-charged nitrilotriacetic matrix (see Section 2). The recombinant MEs from both leishmanial species were expressed in good yields $(\cong 1.3 \mathrm{mg}$ per liter of culture) and showed to be homogeneous in SDS-PAGE under denaturing conditions. The visualized protein bands exhibited apparent molecular masses nearby $65 \mathrm{kDa}$, which closely matched the values predicted from the coding sequences (Lmex_ME-61, $62.6 \mathrm{kDa}$; Lmj_ME-70, 63.4), (Fig. 2A). Moreover, in gel-filtration chromatography under native conditions, both recombinant enzymes eluted in a single symmetric peak, and the elution volume corresponded to a protein of approximately $130 \mathrm{kDa}$ (Fig. 2B). This value fit in well with a homodimeric organization of both leishmanial MEs. The recombinant MEs differed in their protein stability, Lmex_ME-61 remained soluble in the elution buffer for several days, whereas Lmj_ME-70 exhibited a remarkable trend towards aggregation unless desalted in $75 \mathrm{mM}$ TEA buffer, $\mathrm{pH}$ 7.4. The potential influence of the $6 \mathrm{xHis}$ extension at the $\mathrm{N}$-terminus on the solubility of the recombinant MEs could not be examined given that both enzymes were notably more prone to precipitate in the varied thrombin digestion conditions assayed. On the other hand, the recombinant enzymes exhibited no immunological cross-reactivity when examined by Western blot analysis using the specific mouse antisera raised against Lmex_ME-61 and Lmj_ME-70, respectively (Fig. 2C).

\subsubsection{Kinetic properties of the recombinant Lmj_ME-70 and Lmex_ME-61}

Both Lmj_ME-70 and Lmex_ME-61 showed to be functional and specifically catalyzed the reduction of $\mathrm{NADP}^{+}$. In contrast, neither Lmj_ME-70 nor Lmex_ME-61 used NAD ${ }^{+}$as alternative coenzyme, even at $\mathrm{mM}$ concentrations. Besides, unexpectedly the recombinant leishmanial isozymes were inoperative when $\mathrm{Mn}^{2+}$ was substituted by $\mathrm{Mg}^{2+}$ as divalent cation cofactor. Both MEs performed the oxidative decarboxylation of malate notably more efficiently than pyruvate carboxylation. Although Lmj_ME-70 and Lmex_ME-61 were active in a pH range of 7.0-9.0, the two enzymes exhibited the highest catalytic performance at a relatively narrow $\mathrm{pH}$ range (7.0-8.0). Thus, for activity measurements, $75 \mathrm{mM}$ TEA buffer, $\mathrm{pH}$ 7.4, was selected. When malate and $\mathrm{NADP}^{+}$were assayed as substrates, Lmj_ME-70 and Lmex_ME-61 displayed equivalent specific activities $\left(\cong 100 \mathrm{U}^{-\mathrm{mg}^{-1}}\right)$. To further characterize the ME isozymes, the kinetic parameters for malate, $\mathrm{NADP}^{+}$, pyruvate and $\mathrm{Mn}^{2+}$ were determined as described in Section 2. Both leishmanial MEs exhibited equivalent kinetic parameters
A

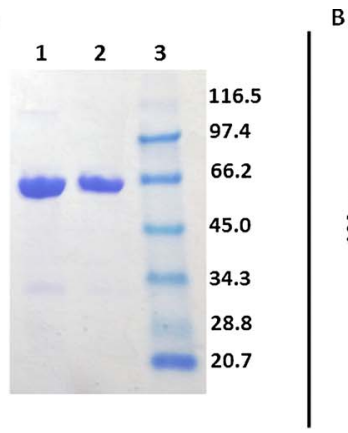

B

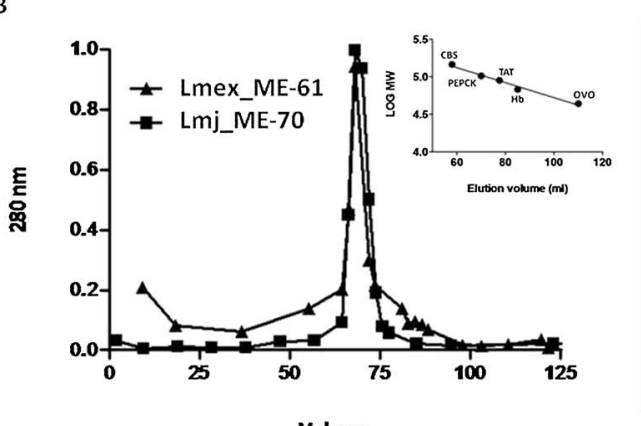

2

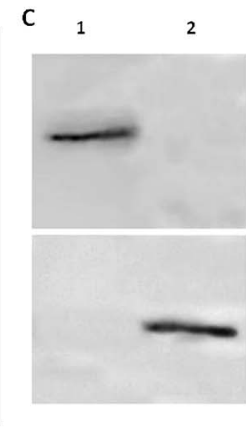

Fig. 2. Heterologous expression and purification of Lmj_ME-70 and Lmex_ME-61. (A) The recombinant 6xHis-tagged Lmj_ME-70 and Lmex_ME-61 were purified as described in the Materials and Methods section. Each of the recombinant proteins $(8 \mu \mathrm{g})$ were subjected to SDS-PAGE in $8.5 \%$ acrylamide gels and visualized by Coomassie Blue staining. Lane 1 , Lmex_ME-61; Lane 2, Lmj_ME-70; Lane 3, molecular mass standards. The mass values of the protein markers are given in $\mathrm{kDa}$ and shown on the right side of the panel. (B) Gel-filtration chromatography under native conditions of Lmj_ME-70 and Lmex_ME-61 was performed as described in the Materials and methods section. The inset shows the standard curve for molecular masses; the proteins used as molecular

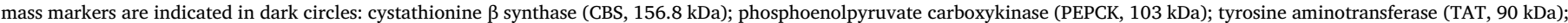

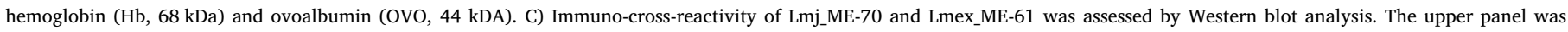

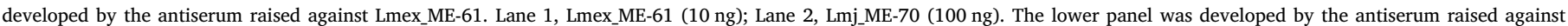

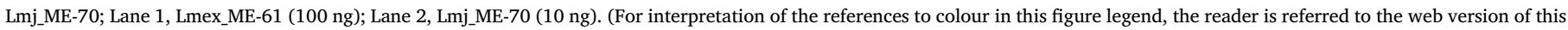
article.) 
Table 1

Apparent kinetic parameters of the ME isozymes from L. major and $L$. mexicana

\begin{tabular}{|c|c|c|c|c|}
\hline Enzyme & Substrate & $K_{\mathrm{m}}(\mathrm{mM})$ & $k_{\text {cat }}\left(\mathrm{s}^{-1}\right)$ & $k_{\text {cat }} / K_{\mathrm{m}}\left(\mathrm{M}^{-1} \mathrm{~s}^{-1}\right)$ \\
\hline \multicolumn{5}{|c|}{ Malate oxidative decarboxylation } \\
\hline & Malate & $0.44 \pm 0.05$ & $113.63 \pm 2.19$ & $(2.58 \pm 0.02) \times 10^{5}$ \\
\hline Lmj_ME-70 & NADP & $0.04 \pm 0.001$ & $119.89 \pm 3.23$ & $(3.00 \pm 0.03) \times 10^{6}$ \\
\hline & $\mathrm{Mn}^{2+}$ & $0.0092 \pm 0.0006$ & $109 \pm 1$ & $(1.18 \pm 0.18) \times 10^{7}$ \\
\hline \multirow{3}{*}{ Lmex_ME-61 } & Malate & $0.65 \pm 0.23$ & $122.23 \pm 7.37$ & $(1.88 \pm 0.06) \times 10^{5}$ \\
\hline & NADP & $0.023 \pm 0.002$ & $106.93 \pm 4.19$ & $(4.65 \pm 0.04) \times 10^{6}$ \\
\hline & $\mathrm{Mn}^{2+}$ & $0.020 \pm 0.001$ & $123.25 \pm 0.65$ & $(6.16 \pm 0.01) \times 10^{6}$ \\
\hline \multicolumn{5}{|c|}{ Pyruvate reductive carboxylation } \\
\hline Lmj_ME-70 & Pyruvate & $2.44 \pm 0.4$ & $18.17 \pm 1.04$ & $(7.44 \pm 0.06) \times 10^{3}$ \\
\hline Lmex_ME-61 & Pyruvate & $11.14 \pm 2.09$ & $64.68 \pm 15.86$ & $(5.81 \pm 0.25) \times 10^{3}$ \\
\hline
\end{tabular}

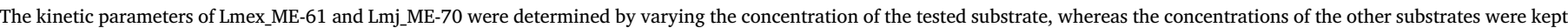

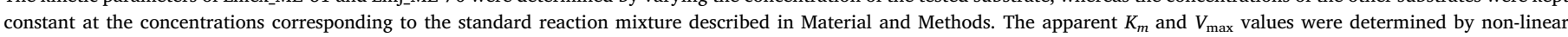

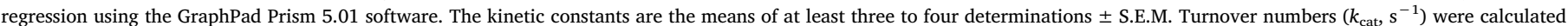
using the molecular mass of one subunit of each ME isozyme.

(Table 1), and displayed typical hyperbolic curves when assayed in the direction of the oxidative decarboxylation of malate (Fig. 3) or pyruvate carboxylation (not shown).

Since it has been well-established that MEs from certain sources are allosterically regulated, different metabolic intermediates were tested to examine whether they affect the activities of Lmj_ME-70 and Lmex_ME-61. Among the intermediates tested, only L-aspartate exhibited the ability to increase the activity of Lmex_ME-61, and none of them displayed any effect on the catalytic competence of Lmex_ME-70. Half maximal activation of Lmex_ME-61 was observed when L-aspartate was added to the reaction mixture at a concentration of $70 \mu \mathrm{M}$ (not shown). To further examine the activation mechanism of L-aspartate, the apparent kinetic parameters towards malate and $\mathrm{NADP}^{+}$were determined in the presence of $5 \mathrm{mM}$ of this amino acid (Fig. 3A and B). The activation effect resulted in a decrease of the apparent $K_{\mathrm{m}}$ value towards malate (in about 6 -fold) in addition to a mild but significant increase in the reaction rate of Lmex_ME-61. Therefore, in the presence of L-aspartate, the catalytic efficiency $\left(k_{\text {cat }} / K_{\mathrm{m}}{ }^{\text {(NADP,malate) }}\right)$ of Lmex_ME61 for NADPH production was increased nearly 10-fold (Fig. 3, panel C).

\subsection{Homology modeling of Lmex_ME-61 and Lmj_ME-70}

Despite the fact that Lmex_ME-61 and Lmj_ME-70 specifically catalyze the production of NADPH, homology blast search revealed that the leishmanial ME isoforms exhibited around $40 \%$ sequence identity with NAD- and NADP-linked homologs from plants, bacteria and eukaryotic counterparts. The sequence stretches containing the predominant determinants of cofactor specificity as well as the typical second motif of the dinucleotide-binding signature of MEs exhibited a lower degree of identity when the leishmanial homologs were compared with the NADP- and NAD(P)-dependent MEs from higher eukaryotes (for clarity see Fig. 1, Supplementary Material). When ConSurf was used as a tool to discriminate between the conservation caused by a short evolutionary time and genuine sequence conservation, the outcomes revealed that the residues known to play critical roles in catalysis and structural stability were among those which displayed the highest degree of conservation among leishmanial homologs such as Lmex_ME61 and Lmj_ME-70. By contrast, the amino acids known to represent the main determinants of coenzyme specificity (sequence stretch 304-356) as well as those of the C-terminus exhibited a significant degree of variability as compared with the 150 closest homologs (Fig. 2A and B, Supplementary Material). On the other hand, when the conservation grades were projected onto the molecular surface of Lmex_ME-61 and Lmj_ME-70, respectively, the areas corresponding to the coenzyme binding pockets exhibited the highest degree of conservation (Fig. 3, Supplementary Material). Subsequently, Lmex_ME-61 and Lmj_ME-70 were selected as the representative members of each of the two isoforms present in Leishmania parasites for a structure-based protein sequence alignment by using the crystal structures of the ligand complexed forms of pigeon liver ME-NADP and human mitochondrial ME-NAD(P) as templates (Fig. 4). To facilitate comparative analysis, as suggested by Yang et al., residues were numbered according to the structural equivalence between the human m-NAD(P)-ME and the cytosolic pigeon ME, and thus the first methionine of the pigeon enzyme is referred to as residue 22 [27]. In line with the outcomes of CONSURF analyses,

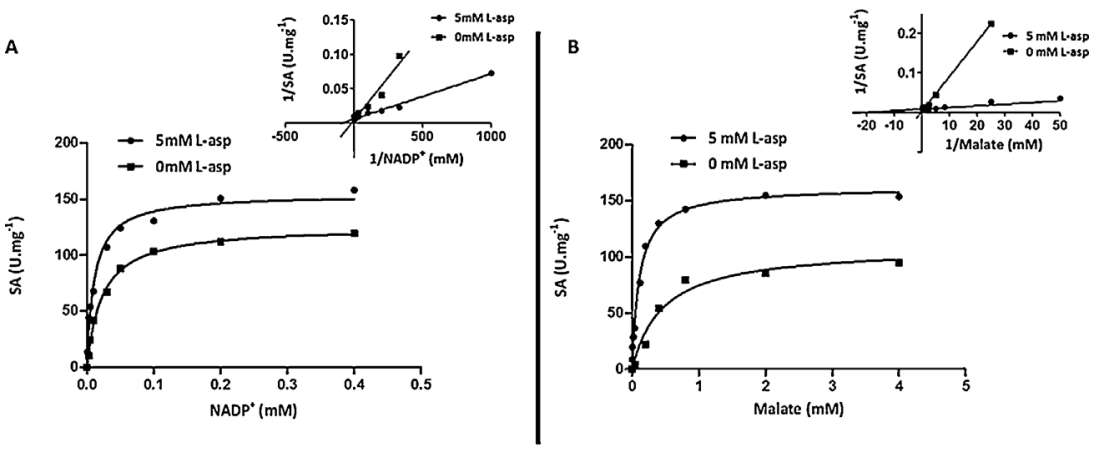

Fig. 3. Allosteric activation of Lmex_ME-61 by L-aspartate. Panels A and B show the Michaelis-Menten plots obtained for $\mathrm{NADP}^{+}$and malate as variable substrates, respectively, in the presence and absence of L-aspartate. The corresponding Lineweaver-Burk plots are depicted as insets on the right side of each panel. Panel C shows the $K_{m}$ and $k_{c a t}$ values obtained in the presence of L-aspartate and correlates these data with those described in Table 1.

\begin{tabular}{|ccccccc|}
\hline C & Substrate & $\kappa_{\mathrm{m}}(\mathrm{mM})$ & Fold-change & $k_{\text {cat }}\left(\mathrm{s}^{-1}\right)$ & Fold-increase & $k_{\text {cat }} / K_{\mathrm{m}}\left(\mathrm{M}^{-1} \cdot \mathrm{s}^{-1}\right)$ \\
\hline \multirow{3}{*}{ Lmex_ME-61 } & Malate & $0.11 \pm 0.01$ & 0.25 & $162.10 \pm 3.65$ & 1.35 & $(1.47 \pm 0.04) \times 10^{6}$ \\
& NADP & $0.01 \pm 0.001$ & 0.43 & $154.20 \pm 4.51$ & 1.35 & $(1.54 \pm 0.04) \times 10^{7}$ \\
\hline
\end{tabular}


the alignment clearly depicted that all the residues critical for activity or divalent cation binding were strictly conserved in the compared MEs. However, the residues known to be related to cofactor specificity, fumarate binding and the second motif of the dinucleotide-binding signature of MEs $\left({ }^{311}\right.$ GAGEAA $\left.^{316}\right)$ were preserved in a lower degree. Besides, the C-terminal fragment of the leishmanial MEs also displayed a notable lack of sequence relatedness with the higher eukaryotic counterparts (Fig. 4).

To gain a deeper insight into the molecular organization of leishmanial MEs, 3-D homology models of Lmex_ME-61 and Lmj_ME-70 were generated. Given that the two leishmanial isozymes functionally characterized in this work specifically catalyzed the reduction of $\mathrm{NADP}^{+}$, the crystal structure of NADP-dependent ME from pigeon in quaternary complex with $\mathrm{NADP}^{+}, \mathrm{Mn}^{2+}$, and oxalate (PBD 1GQ2) was used as template. Once the selected models of Lmex_ME-61 and Lmj_ME-70 were subjected to refinement processes, PROCHECK Ramachandran plots showed that $\gg>95 \%$ of the residues fell within the fully allowed regions in both enzymes models (Fig. 4, Supplementary Material). In parallel, Verify 3D revealed good compatibility between the atomic model (3D) and the target amino acid sequence (1D) (Fig. 4, Supplementary Material). As first outcome, the homology modeling analyses indicated that Lmex_ME-61 and Lmj_ME-
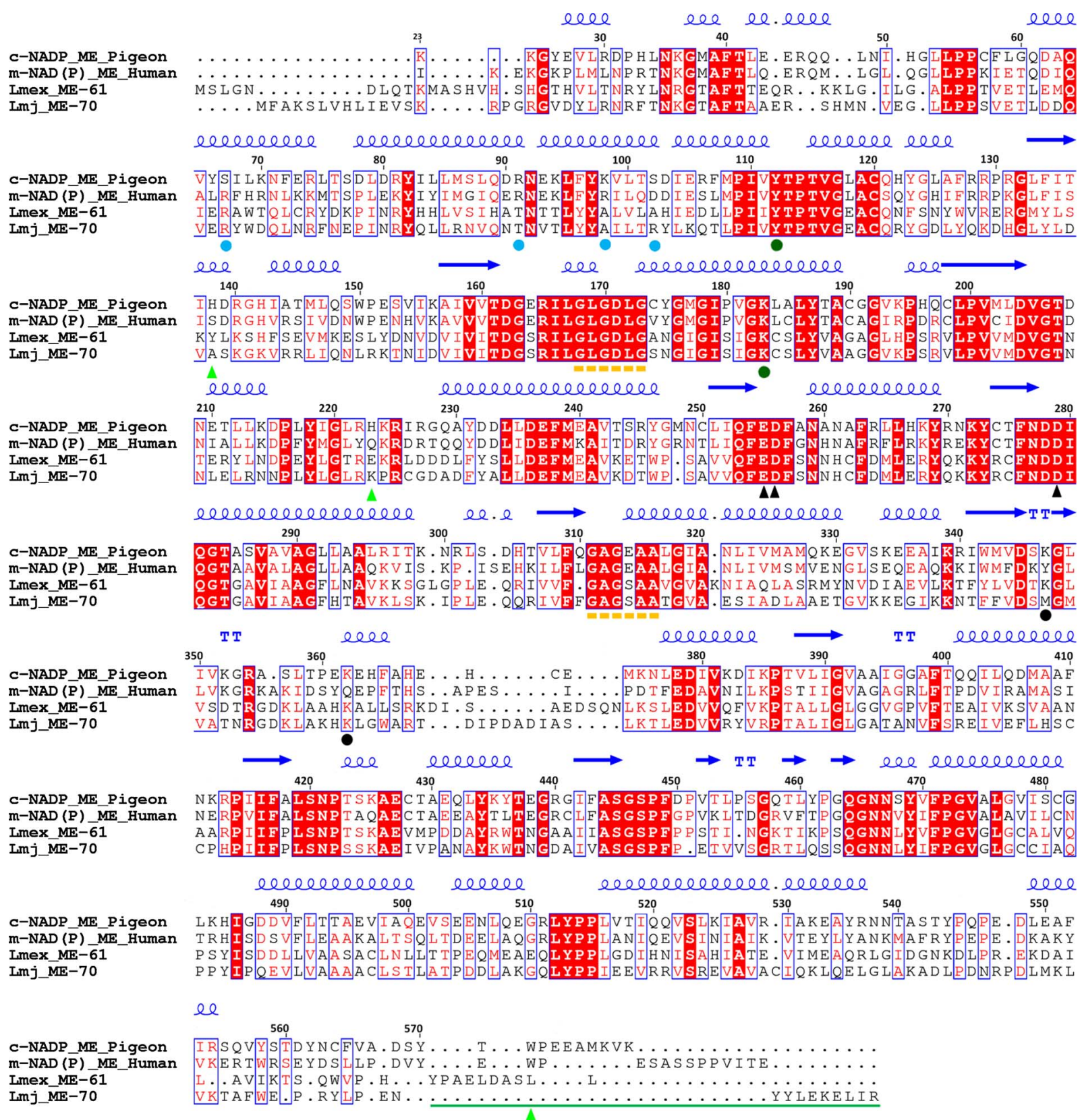

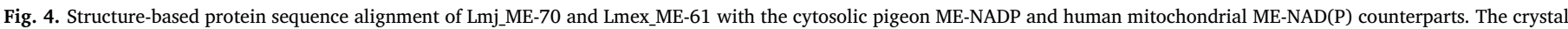

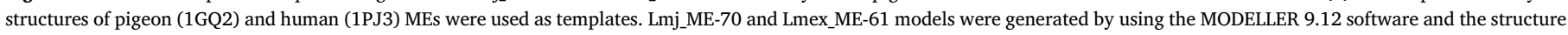

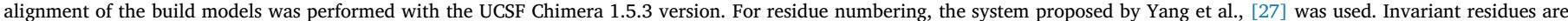

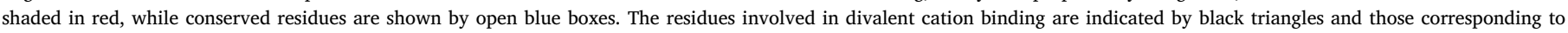

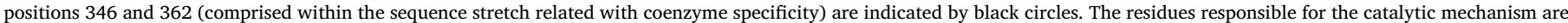

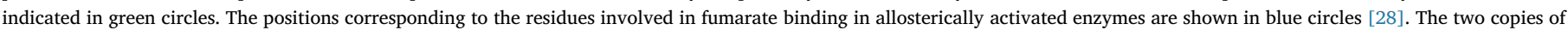

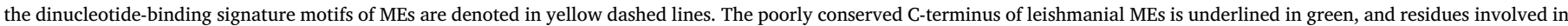

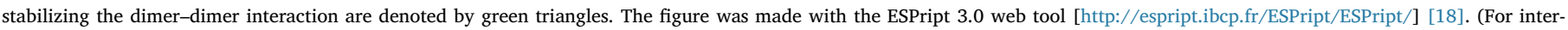
pretation of the references to colour in this figure legend, the reader is referred to the web version of this article.) 
70 might differ in their secondary structures. In Lmj_ME-70, a disulfide bridge between C493 and C526 was predicted (Fig. 5, Supplementary Material). Notably, the cysteine residues involved in this potential disulfide bridge are strictly conserved among the leishmanial homologs of Lmj_ME-70, however C526 is absent in Lmex_ME-61 counterparts. In line with CONSURF outputs, only the residue equivalent to C526 was found among the highly conserved residues throughout the members of ME family (see Fig. 2B Supplementary Material). This finding suggested that the predicted disulfide bridge could represent a particular feature of Lmj_ME-70 and its closest counterparts.

Given that structure quality factors indicated that the models build for Lmex_ME-61 and Lmj_ME-70 were statistically reliable [29], the modeled enzymes were further analyzed to explore plausible differences in the surface charge pattern distribution as well as visualizing the residues potentially involved in $\mathrm{NADP}^{+}$binding. To address the first issue, the electrostatic potentials of the overall surfaces of one subunit of each leishmanial MEs were examined, and attention was focused on the areas surrounding the coenzyme binding pockets of Lmex_ME-61 and Lmj_ME-70. The build models evidenced that the molecular surface of Lmex_ME-61 presented a lower abundance of positively charged side chains as compared with Lmj_ME-70. An equivalent trend was also observed in the areas surrounding the $\mathrm{NADP}^{+}$ binding pockets of Lmj_ME-70 and Lmex_ME-61 (Fig. 5, panels A, B, C and $\mathrm{D}$ ). The differences observed in the surface electrostatic potentials fit in well with the higher pI value predicted for Lmj_ME-70 towards Lmex_ME-61, as well as with the lack of immunological cross-reactivity between both isozymes.

Moreover, when the build models were subjected to analysis using automated integrated platform for structure-based multiple-level protein function predictions such as COFACTOR, Lmex_ME-61 and Lmj_ME-70 exhibited the highest global fold similarities with the mitochondrial ME-NAD (100sA) and ME-NAD(P) (1eflA) from the nematode Ascaris suum and man, respectively, in addition to the human cytosolic ME-NADP (2aw5B). However, when focusing on the coenzyme binding sites, the pigeon cytosolic ME-NADP was the best ranked homolog for both leishmanial ME isozymes. The outcomes of these analyses indicated that the confidence scores predicting the $\mathrm{NADP}^{+}$ binding pockets were lower than those corresponding to the overall structure similarities (the scores validating the predicted results are summarized in Fig. 6A and B, Supplementary Material).

On the other hand, the topological survey of the coenzyme binding pockets of Lmj_ME-70 and Lmex_ME-61 revealed that the side chains of T340 and S339 as well as K356 and K355 from Lmex_ME-61 and Lmj_ME-70, respectively, clearly displayed the expected orientations for establishing hydrogen bonding with the 2'-phosphate group of the coenzyme (Fig. 6, , panels A and B). Moreover, the side chains of K341 (Lmex_ME-61) and M340 (Lmj_ME-70) displayed analogous spatial orientation, pointing outside the $\mathrm{NADP}^{+}$binding pockets of both leishmanial MEs. Also, in the modeled coenzyme binding pockets of Lmj_ME-70 and Lmex_ME-61, the side chain of D339 (Lmex_ME-61) and D338 (Lmj_ME-70) were observed establishing an ion-pair interaction with R348 and R347, respectively. In all $\mathrm{NADP}^{+}$-dependent MEs, this ion-pair is essential to avoid charge repulsion between the $\beta$-carboxylate group of this aspartate residue and the 2'-phosphate group of the coenzyme (Fig. 6 panels A and B). Lastly, the ligplot graphs allowed gaining a better insight into the residues lining the coenzyme binding pockets of both leishmanial MEs, identifying those residues involved in direct or indirect contacts with the coenzyme. These graphs also provided information about the distances of the main hydrogen bond interactions among the side chains of the amino acids lining the NADP ${ }^{+}$ binding pocket and the nicotinamide and adenine moieties of the coenzyme (Fig. 6, panels $\mathrm{C}$ and $\mathrm{D}$ ). In addition, these diagrams also revealed that S305, the residue at the position of E314 within the second dinucleotide-binding signature motif of MEs (Fig. 4), and its contiguous alanine residues exhibited a close spatial allocation with respect to the coenzyme bound in the pocket area of Lmex_ME-61 and Lmj_ME-70 (Fig. 6, panels C and D).

\subsection{Evaluation of the expression patterns of the ME isoforms in promastigotes from Leishmania parasites}

Keeping in mind that the expression of Lmex_ME-70 has been confirmed by proteomic analysis of L. major promastigotes [30], we next examined whether either of the two ME isozymes was preferentially expressed in those species that displayed the two gene copies. To this end, cell-free extracts derived from promastigotes of $L$. mexicana, $L$. amazonensis and $L$. braziliensis were examined by Western blot analysis. These assays evidenced that Lmex_ME-61 and its closest homologs appeared to be predominantly expressed in the selected species. By contrast, the ME-70 counterparts could not be detected in the crude extracts from the assessed Leishmania parasites (Fig. 7).

\section{Discussion}

Our results revealed that Leishmania parasites exhibit two functional ME isozymes highly specific towards $\mathrm{NADP}^{+}$. However, both enzymes behave differently in response to allosteric activators, are predicted not to display identical secondary structures and appear to exhibit a
A

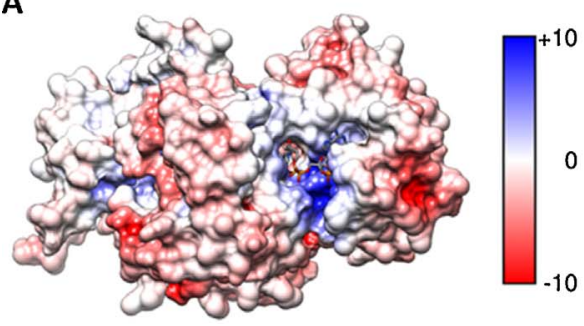

C

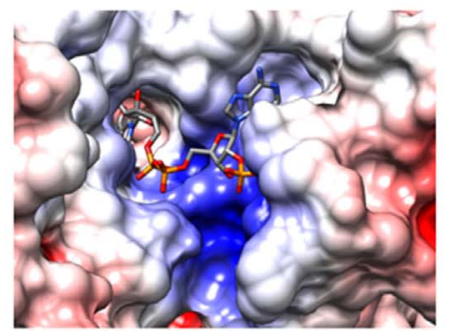

B

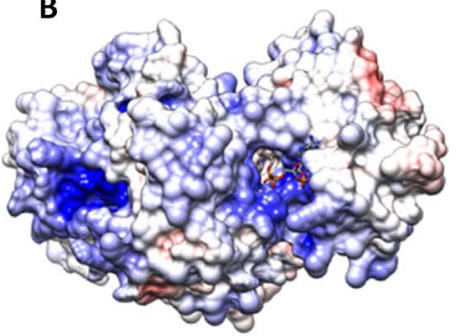

D

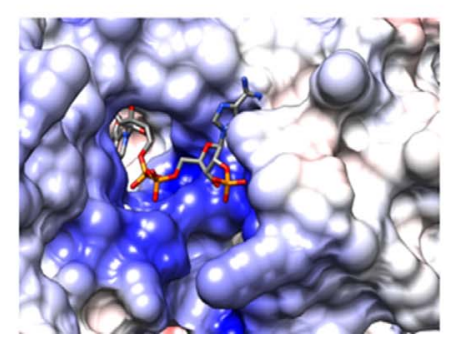

Fig. 5. Comparison of the charge pattern distribution on the molecular surfaces of Lmex_ME-61 and Lmj_ME-70. The electrostatic potentials of the molecular areas are colored as follows: negative and positive charges are represented in red and blue, respectively, while areas with neutral charges are indicated in white. Panels A and B depict one subunit surface of Lmex ME-61 and Lmj_ME-70, respectively. Panels $C$ and $D$ show a zoom view of the areas surrounding the NADP $^{+}$binding pockets of Lmex_ME-61 and Lmj_ME-70, respectively. The figures were generated with UCSF Chimera 1.11.2 (https://www. cgl.ucsf.edu/chimera/). (For interpretation of the references to colour in this figure legend, the reader is referred to the web version of this article.) 
A

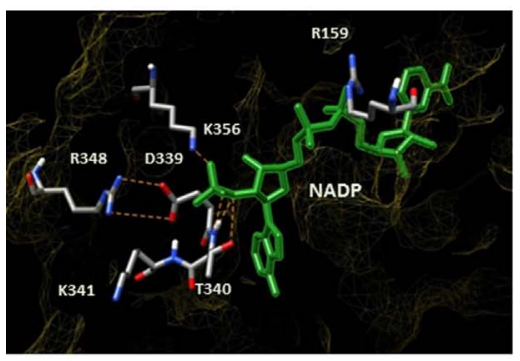

C

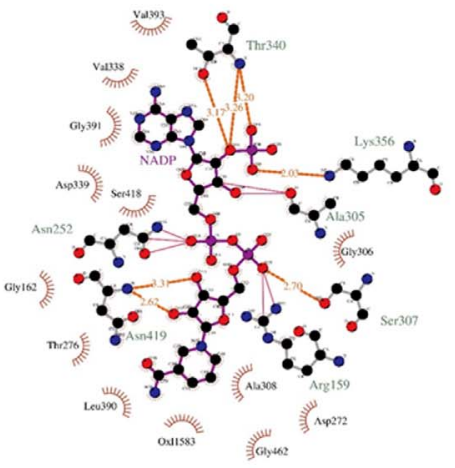

B

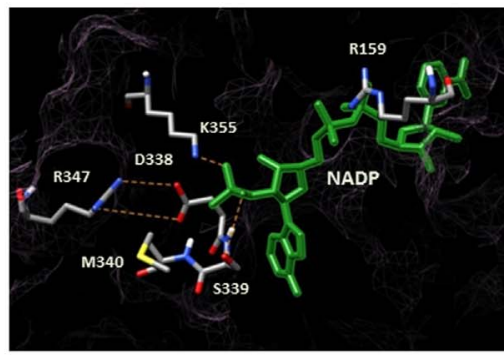

D

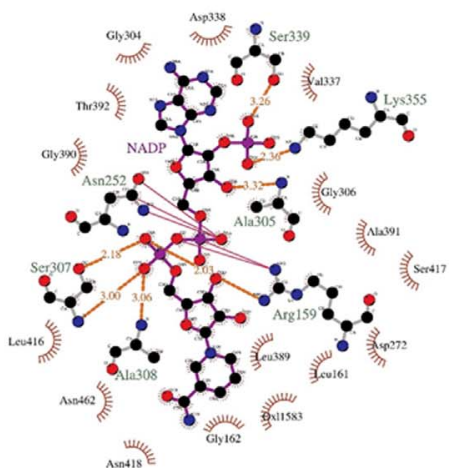

Fig. 6. NADP ${ }^{+}$binding pockets of modeled Lmex_ME-61 and Lmj_ME70. Panels A and B show the amino acid residues interacting with the coenzyme in Lmex_ME-61 and Lmj_ME-70, respectively. The molecular structure of $\mathrm{NADP}^{+}$is shown in green sticks. The hydrogen bonding of the residues at positions 346 and 362 according structural equivalence between the human m-NAD(P)-ME and the cytosolic pigeon ME [27], T340, S339 and K356 and K355, in Lmex_ME-61 and Lmj_ME-70, respectively with $2^{\prime}$-phosphate of NADP ${ }^{+}$are shown in yellow dashed lines. Also, the ion pair interaction between R354 and D345 (R339 and D348 in Lmex_ME-61, and D338 and R347 in Lmj_ME-70, respectively) are represented in yellow dashed lines. Panels $\mathrm{C}$ and $\mathrm{D}$ depict the LIGPLOT diagrams and illustrate the distances of the main hydrogen bond interactions established between the side chains and the coenzyme in Lmex_ME-61 and Lmj_ME-70, respectively. Spoked arcs represent hydrophobic contacts. Models were built and figures were generated as described in the Materials and methods section. (For interpretation of the references to colour in this figure legend, the reader is referred to the web version of this article.) species-specific expression pattern. The two ME isozymes from Leishmania parasites are distributed in two different clades of the phylogenetic tree constructed with $21 \mathrm{ME}$ sequences from the order Kinetoplastea (Fig. 1). Although the set of leishmanial MEs allocated in

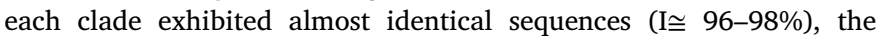
counterparts from the different clades revealed notably lower related-

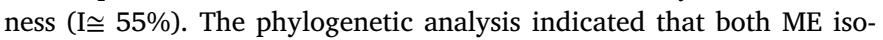
zymes very likely derive from organisms which probably existed as freeliving organisms before the origin of parasitism [31]. Therefore, with the exception of L. major, LmxM.24.0761 and $L m x M .24 .0770$ as well as their closest relatives are likely to represent Kinetoplastea genes that were retained after the origin of parasitism in the Leishmania species. At variance, $L$. major could afford to recently lose this gene (the copy corresponding to LmxM.24.0761 and its closest homologs in other species) for reasons still to be determined.

The two leishmanial ME isoforms exhibited equivalent specific activities, our kinetic studies showed that while in the presence of L-aspartate the catalytic performance of Lmex_ME-61 is notably higher, that of Lmj_ME-70 remains unaffected. Besides, as compared with trypanosomial homologs, Lmex_ME-61 resembles the cytosolic isoform of $T$. cruzi ME, which also is allosterically activated by L-aspartate [26]. Lmex_ME-61 displays a typical Michaelis-Menten behavior and exhibits no signs of cooperativity in the absence of L-aspartate. This fact clearly differentiates Lmex_ME-61 from the human mitochondrial ME-NAD(P) and the cytosolic ME-NADP. The former is activated by fumarate and exhibits a cooperative behavior towards malate in the absence of its allosteric activator, while the latter is unresponsive to allosteric activators [3,32]. Moreover, as summarized in Table 2, Lmj_ME-70 and Lmex_ME-61 exhibited higher $K_{\mathrm{m}}$ values towards the coenzyme while those for malate were noticeably lower. In addition, unlike most MEs, the leishmanial isozymes appear to specifically depend on $\mathrm{Mn}^{2+}$ as divalent cation cofactor, being inactive in the presence of $\mathrm{Mg}^{2+}$, and the catalytic rates of both leishmanial MEs nearly triplicate the values of the human counterparts [32]. Hence, in terms of catalytic mechanism, both leishmanial MEs seem to behave differently than the mammalian counterparts.

Also, Lmex_ME-61 and Lmj_ME-70 appear to differ from the great majority of MEs in that, in native conditions, the isozymes from this pathogen exhibit a dimeric organization. The correlation between the catalytic properties and quaternary structure organization of MEs is still unclear. Indeed, it has been shown that dimers and tetramers of human MEs are equally active [33]. The hydrophobic interactions established by the residues of the C-terminal tail have been recognized as the main driving force for subunit assembly in the mammalian enzymes. However, additional hydrogen bonding and ionic interactions also markedly contribute to the oligomeric organization of MEs [34]. Outstandingly, W572, which is known to play a crucial role in the tetrameric organization of MEs [35], is among the not conserved residues in the C-
A

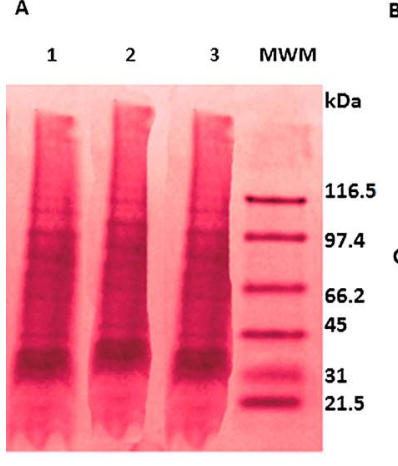

B
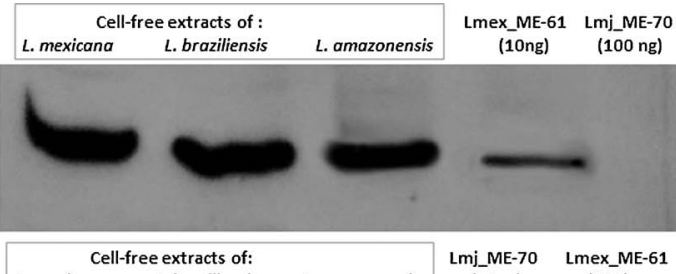

\begin{tabular}{|ccc|cc}
\multicolumn{2}{c|}{ Cell-free extracts of: } & & Lmj_ME-70 & Lmex_ME-61 \\
L. mexicana & L. braziliensis & L. amazonensis & (10ng) & (100) $\mathrm{ng}$
\end{tabular}

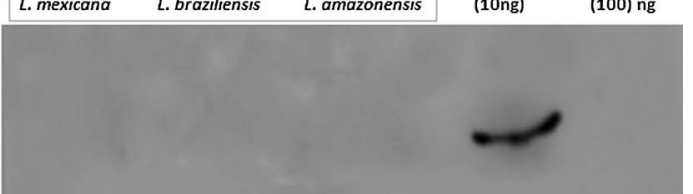

Fig. 7. Expression patterns of the ME isoforms in promastigotes of Leishmania parasites. Identical number of L. mexicana, L. braziliensis, and $L$. amazonensis promastigotes $\left(10 \times 10^{6}\right.$ cells $)$ were suspended in equal volumes of SDS-PAGE sample buffer, and the total proteins of the lysates obtained were resolved in $8.5 \%$ acrylamide gel and subjected to Western blot analysis as described in Materials and Methods. Panel A shows the nitrocellulose membrane stained with Ponceau-S. Lane 1, L. mexicana; Lane 2, L. braziliensis, Lane 3 L. amazonensis; and MWM, molecular weight standards. The mass values of the protein markers are given in $\mathrm{kDa}$ and shown on the right side of the panel. Panels B and C illustrate the immunocomplexes detected when the cell-free extracts and both recombinant MEs (Lmex ME-61 and Lmj_ME-70) were tested with the antisera raised against Lmex_ME-61 (Panel B) and Lmj_ME-70 (Panel C), respectively. (For interpretation of the references to colour in this figure legend, the reader is referred to the web version of this article.) 
Table 2

Comparison of the kinetic properties of Lmj_ME-70 and Lmex_ME-61 with human NAD(P) and NADP dependent orthologs.

\begin{tabular}{|c|c|c|c|c|c|}
\hline Enzyme & $K_{\mathrm{m}}$ malate $(\mathrm{mM})$ & $K_{\mathrm{m}}^{\mathrm{NADP}}(\mu \mathrm{M})$ & ${ }^{\mathrm{a}} k_{\text {cat }}\left(\mathrm{s}^{-1}\right)$ & Allosteric activator & Cooperativity towards malate \\
\hline Lmj_ME-70 & $0.44 \pm 0.05$ & $40 \pm 1$ & $116.8 \pm 2$ & $(-)$ & $(-)$ \\
\hline Lmex_ME-61 & $0.65 \pm 0.23$ & $23 \pm 2$ & $114.6 \pm 3$ & Aspartate $(-)$ & $(-)$ \\
\hline Lmex_ME-61 & $0.11 \pm 0.01$ & $10 \pm 1$ & $160 \pm 3$ & Aspartate $(+)$ & $(-)$ \\
\hline${ }^{d}$ hcit_ME-NADP & $1.2 \pm 0.06$ & $1.9 \pm 0.2$ & $30 \pm 0.2$ & $(-)$ & $(-)$ \\
\hline${ }^{\mathrm{d}}$ hmit_ME-NAD(P) & $15.6 \pm 0.6^{\mathrm{b}}$ & $1.4 \pm 0.1^{\mathrm{c}}$ & $43 \pm 1.0$ & Fumarate $(-)$ & $(+)$ \\
\hline${ }^{d}$ hmit_ME-NAD(P) & $4.6 \pm 0.2^{\mathrm{b}}$ & $0.3 \pm 0.02^{\mathrm{c}}$ & $55 \pm 0.6$ & Fumarate $(+)$ & $(+)$ \\
\hline
\end{tabular}

${ }^{\text {a }}$ The $k_{\text {cat }}$ values represent the catalytic rate constants of an active site.

b This value corresponds to $K_{0.5}$ for malate.

c This value corresponds to the $K_{\mathrm{m}}$ towards $\mathrm{NAD}^{+}$.

${ }^{d}$ Kinetic parameters were adapted from Hsieh et al., 2014 [32].

terminus of leishmanial MEs (Fig. 4). Therefore, as compared with higher eukaryotic counterparts, it is tempting to speculate that the absence of the two histidine residues (H138 and H223) known to contribute to the homotetrameric structure as well as the poorly conserved C-terminal tail could lead to weaker contacts at the dimer interfaces and to a less favored dimer-dimer association in leishmanial MEs.

The bioinformatic assessments showed that the two leishmanial MEs exhibited a highly conserved overall structural topology (Fig. 6A Supplementary Material). However, lower confidence scores were obtained for the coenzyme binding pockets of both enzymes (Fig. 6B Supplementary Material). These outputs not only fit in well with the conserved folding pattern among MEs [1], but also with the particularly poor similarity within the sequence stretches known to contain the predominant determinants of cofactor specificity. Besides, the comparative analysis of the 3-D models of Lmex_ME-61 and Lmj_ME-70 depicted that both leishmanial isozymes not only could exhibit differences in their secondary structures but also notably differ in their surface charge distribution (Fig. 5). In parallel, as compared with Lmj_ME70 , the area surrounding the coenzyme binding pocket of Lmex_ME-61 also distinguishes significantly by the lower abundance of positive charges. Given that K347 localizes in this region and is highly conserved in the coenzyme binding site of $\mathrm{NADP}^{+}$-dependent MEs, but substituted by varied residues in $\mathrm{NAD}^{+}$- dependent MEs (Fig. 1, Supplementary Material), its substitution by methionine in Lmj_ME-70 (see Fig. 4) attracted our attention. For instance, at position 347, MEs such as the human mitochondrial ME-NAD $(\mathrm{P})^{+}$or the Ascaris suum $\mathrm{NAD}^{+}$-dependent homolog, exhibit a tyrosine and aspartic residue, respectively (Fig. 1 Supplementary material). However, the functional characterization of Lmj_ME-70 indicated that, like in other $\mathrm{NADP}^{+}$. linked MEs, the residue at this position is not critical for NADP ${ }^{+}$ binding even though strictly conserved [36]; thus, its potential role - if any- remains to be disclosed. Despite these differences, homology modeling showed that the few residues known to play critical roles in $\mathrm{NADP}^{+}$binding are well conserved in the binding pockets of both leishmanial isozymes. Moreover, despite the substitutions in the highly conserved dinucleotide-binding signature motif of MEs $\left({ }^{311}\right.$ GAGEAA ${ }^{316}$, Fig. 4), the residues comprised in this sequence stretch are observed lining the modeled $\mathrm{NADP}^{+}$binding pockets of both leishmanial isozymes (Fig. 6). Moreover, in line with the lack of allosteric activation by fumarate, only R67 of the four residues (R67, R91, R98 and D102) known to play critical roles in this ligand binding is strictly conserved in the leishmanial MEs (Fig. 4).

It could be argued that the differences in the kinetic properties and protein surface charge distribution between Lmex_ME-61 and Lmj_ME70 might imply potential differences in biological roles, subcellular localization or in in vivo protein-protein interactions. These findings fit in well with recent reports which point out that the metabolic processes might be not necessarily strictly conserved among the different Leishmania species. Indeed, recent comparative analysis of metabolomes from promastigotes of several Leishmania parasites (L. major, $L$. mexicana, and L. donovani) has shown significant variations among the intracellular concentration of different amino acids and metabolic intermediates [37]. It is tempting to speculate that, in species that express the closest homologs of Lmex_ME-61, L-aspartate could boost the conversion of malate into pyruvate. The higher trend towards pyruvate formation could not only feed the Krebs cycle but also contribute to an increase in the production of NADPH, which might be required to neutralize the metabolic stress in addition to supplying the so critical reducing equivalents on which these pathogens depend for survival.

In brief, our results provide evidence that leishmanial MEs display features that clearly distinguish these enzymes from human counterparts. Our findings open up the possibility of structural studies to achieve a more detailed view of the molecular organization of leishmanial MEs, as well as identifying the still undisclosed binding site of $\mathrm{L}^{-}$ aspartate. This knowledge in addition to more comprehensive biochemical studies will shed light on the metabolic roles of MEs in Leishmania parasites.

\section{Acknowledgments}

This work was performed with grant to $\mathrm{CN}$ from Universidad de Buenos Aires (UBACYT 20100100618). CN and PP are member of the Research Career from CONICET; LG and MHS were PhD students supported by CONICET and UBA respectively; AEL was a PhD student supported by CONICET and currently a member of the Research Career from CONICET; EFRM is supported by a PhD fellowship from CAPES (Coordenação de Aperfeiçoamento do Pessoal de Ensino Superior).

\section{Appendix A. Supplementary data}

Supplementary data associated with this article can be found, in the online version, at http://dx.doi.org/10.1016/j.molbiopara.2017.11. 001.

\section{References}

[1] G.G. Chang, L. Tong, Structure and function of malic enzymes: a new class of oxidative decarboxylases, Biochemistry 42 (2003) 12721-12733.

[2] J.K. Teller, L.A. Fahien, J.W. Davis, Kinetics and regulation of hepatoma mi tochondrial NAD(P) malic enzyme, J. Biol. Chem. 267 (1992) 10423-10432.

[3] J.Y. Hsieh, G.Y. Liu, G.G. Chang, H.C. Hung, Determinants of the dual cofactor specificity and substrate cooperativity of the human mitochondrial NAD $(\mathrm{P})^{+}$-dependent malic enzyme: functional roles of glutamine 362, J. Biol. Chem. 281 (2006) 23237-23245.

[4] A.E. Leroux, R.L. Krauth-Siegel, Thiol redox biology of trypanosomatids and potential targets for chemotherapy, Mol. Biochem. Parasitol. 206 (2016) 67-74.

[5] R.L. Krauth-Siegel, M.A. Comini, Redox control in trypanosomatids: parasitic protozoa with trypanothione-based thiol metabolism, Biochim. Biophys. Acta. 1780 (2008) 236-248.

[6] S.H. Lee, J.L. Stephens, P.T. Englund, A fatty-acid synthesis mechanism specialized for parasitism, Nat. Rev. Microbiol. 5 (2007) 287-297.

[7] B. Mittra, N.W. Andrews, IRONy OF FATE: role of iron-mediated ROS in Leishmania differentiation, Trends Parasitol. 10 (2013) 489-496.

[8] M.A. Comini, C. Ortíz, J.J. Cazzulo, T. Jäger, O. Koch, L. Flohé (Eds.), Drug Targets in Trypanosomal and Leishmanial Pentose Phosphate Pathway in Trypanosomatid 
Diseases: Molecular Routes to Drug Discovery, Wiley-VCH Verlag GmbH \& Co. KgaA, Weinheim, Germany, 2013, pp. 297-313.

[9] M.J. McConville, E.C. Saunders, J. Kloehn, M.J. Dagley, Leishmania carbon metabolism in the macrophage phagolysosome- feast or famine? F1000 Research 4 (2015) 938, http://dx.doi.org/10.12688/f1000research.6724.1.

[10] J. Kovářová, M.P. Barrett, The Pentose Phosphate Pathway in Parasitic Trypanosomatids, Trends Parasitol. S1471-4922 (2016) 30013-30017.

[11] S. Allmann, P. Morand, C. Ebikeme, L. Gales, M. Biran, J. Hubert, A. Brennand, M. Mazet, J.M. Franconi, P.A. Michels, J.C. Portais, M. Boshart, F. Bringaud, Cytosolic NADPH homeostasis in glucose-starved procyclic Trypanosoma brucei relies on malic enzyme and the pentose phosphate pathway fed by gluconeogenic flux, J. Biol. Chem. 288 (2013) 18494-18505.

[12] L. Giordana, B. Suárez Mantilla, M. Santana, A. Silber, C. Nowicki, Cystathionine ályase, an enzyme related to the reverse transsulfuration pathway, is functional in Leishmania spp, J. Eukaryot Microbiol. 61 (2014) 204-213.

[13] E. Medina-Acosta, G.A. Cross, Rapid isolation of DNA from trypanosomatid protozoa using a simple 'mini-prep' procedure, Mol. Biochem. Parasitol. 59 (1993) 327-329.

[14] H. Schägger, G. von Jagow, Tricine-sodium dodecyl sulfate-polyacrylamide gel electrophoresis for the separation of proteins in the range from 1 to 100 äkDa, Anal. Biochem 166 (1987) 368-379.

[15] M.M. Bradford, A rapid and sensitive method for the quantitation of micro-gram quantities of protein utilizing the principle of protein-dye binding, Anal. Biochem. 72 (1976) 248-254.

[16] G. Jobb, A. von Haeseler, K. Strimmer, TREEFINDER: a powerful graphical analysis environment for molecular phylogenetics, BMC Evol. Biol. 4 (2004) 18.

[17] A.P. Jackson, M.A. Quail, M. Berriman, Insights into the genome sequence of a freeliving Kinetoplastid: bodo saltans (Kinetoplastida: Euglenozoa), BMC Genomics 9 (2008) 594.

[18] P. Gouet, X. Robert, E. Courcelle, ESPript/ENDscript: extracting and rendering sequence and 3D information from atomic structures of proteins, NucleicAcids Res. 31 (2003) 33203323.

[19] D. Xu, Y. Zhang, Improving the physical realism and structural accuracy of protein models by a two-step atomic-level energy minimization, Biophys. J. 101 (2011) 2525-2534.

[20] R.A. Laskowski, M.W. MacArthur, D.S. Moss, J.M. Thornton, PROCHECK: a program to check the stereochemical quality of protein structures, J. Appl. Cryst. 26 (1993) $283 e 291$.

[21] D. Eisenberg, R. Lüthy, J.U. Bowie, VERIFY3D: assessment of protein models with three-Dimensional profiles, Methods Enzymol. 277 (1997) 396-404.

[22] C. Zhang, P.L. Freddolino, Y. Zhang, COFACTOR: improved protein function prediction by combining structure, sequence and protein-protein interaction information, Nucleic Acids Res. (2017), http://dx.doi.org/10.1093/nar/gkx366.

[23] E.F. Pettersen, T.D. Goddard, C.C. Huang, G.S. Couch, D.M. Greenblatt, E.C. Meng,
T.E. Ferrin, Chimera-a visualization system for exploratory research and analysis, J. Comput. Chem. 13 (2004) 16051612.

[24] R.A. Laskowski, M.B. Swindells, LigPlot: multiple ligand-protein interaction diagrams for drug discovery, J. Chem. Inf Model. 51 (2011) 2778-2786.

[25] H. Ashkenazy, S. Abadi, E. Martz, O. Chay, I. Mayrose, T. Pupko, Ben-Tal N. ConSurf 2016: an improved methodology to estimate and visualize evolutionary conservation in macromolecules, Nucleic Acids Res. 8 (2016) W344-50.

[26] A.E. Leroux, D.A. Maugeri, F.R. Opperdoes, J.J. Cazzulo, C. Nowicki, Comparative studies on the biochemical properties of the malic enzymes from Trypanosoma cruzi and Trypanosoma brucei, FEMS Microbiol. Lett. 314 (2011) 25-33.

[27] Z. Yang, H. Zhang, H.C. Hung, C.C. Kuo, L.C. Tsai, H.S. Yuan, W.Y. Chou, G.G. Chang, L. Tong, Structural studies of the pigeon cytosolic NADP(+)-dependent malic enzyme, Protein Sci. 11 (2002) 332-341.

[28] X. Tao, Z. Yang, L. Tong, Crystal structures of substrate complexes of malic enzyme and insights into the catalytic mechanism, Structure 11 (2003) 1141-1150.

[29] R.W.W. Hooft, C. Sander, G. Vriend, Objectively judging the quality of a protein structure from a Ramachandran plot, Comput. Appl. Biosci. 13 (1997) 425-430.

[30] H. Pawar, S. Renuse, S.N. Khobragade, S. Chavan, G. Sathe, P. Kumar, K.N. Mahale, K. Gore, A. Kulkarni, T. Dixit, R. Raju, T.S. Prasad, H.C. Harsha, M.S. Patole, A. Pandey, Neglected tropical diseases and omics science: proteogenomics analysis of the promastigote stage of Leishmania major parasite, OMICS 8 (2014) 499-512.

[31] A.P. Jackson, T.D. Otto, M. Aslett, S.D. Armstrong, F. Bringaud, A. Schlacht, C. Hartley, M. Sanders, J.M. Wastling, J.B. Dacks, A. Acosta-Serrano, M.C. Field, M.L. Ginger, M. Berriman, Kinetoplastid phylogenomics reveals the evolutionary innovations associated with the origins of parasitism, Curr. Biol. 26 (2016) 161-172.

[32] J.Y. Hsieh, S.Y. Li, M.C. Chen, P.C. Yang, H.Y. Chen, N.L. Chan, J.H. Liu, H.C. Hung, Structural characteristics of the nonallosteric human cytosolic malic enzyme, Biochim. Biophys. Acta 1844 (2014) 1773-1783.

[33] J.-Y. Hsieh, C.-S. Chen, H.-C. Hung, Functional roles of the tetramer organization of malic enzyme, J. Biol. Chem. 284 (2009) 18096-18105.

[34] S. Murugan, H.-C. Hung, Biophysical characterization of the dimer and tetramer interface interactions of the human cytosolic malic enzyme, PLoS One 12 (2012) e50143.

[35] H.C. Chang, G.G. Chang, Involvement of single residue tryptophan 548 in the quaternary structural stability of pigeon cytosolic malic enzyme, J. Biol. Chem. 278 (2003) 23996-24002.

[36] C.C. Kuo, L.C. Tsai, T.Y. Chin, G.G. Chang, W.Y. Chou, Lysine residues 162 and 340 are involved in the catalysis and coenzyme binding of $\mathrm{NADP}\left({ }^{+}\right)$-dependent malic enzyme from pigeon, Biochem. Biophys. Res. Commun. 270 (2000) 821-825.

[37] G.D. Westrop, R.A. Williams, L. Wang, T. Zhang, D.G. Watson, A.M. Silva, G.H. Coombs, Metabolomic analyses of Leishmania reveal multiple species differences and large differences in amino acid metabolism, PLoS One 10 (2015) e0136891. 